\title{
Intake of indigestible carbohydrates influences IgA response and polymeric Ig receptor expression in the rat submandibular gland
}

\author{
Yuko Yamamoto $^{1}$, Masahiro To ${ }^{1}$, Takashi Hayashi ${ }^{1}$, Tomoko Shimizu ${ }^{1}$, Yohei Kamata ${ }^{2}$, Juri Saruta ${ }^{1}$, \\ Toru Takahashi ${ }^{3}$ and Keiichi Tsukinoki ${ }^{1}{ }^{*}$ \\ ${ }^{1}$ Division of Environmental Pathology, Department of Oral Science, Kanagawa Dental University, \\ Graduate School of Dentistry, 82 Inaoka-Cho, Yokosuka, Kanagawa, Japan \\ ${ }^{2}$ Department of Highly Advanced Stomatology, Kanagawa Dental University, Graduate School of Dentistry, \\ 82 Inaoka-Cho, Yokosuka, Kanagawa, Japan \\ ${ }^{3}$ Department of Nutrition and Health Sciences, Fukuoka Women's University, Fukuoka, Japan
}

(Submitted 17 July 2014 - Final revision received 18 February 2015 - Accepted 28 March 2015 - First published online 22 May 2015)

\section{Abstract}

Secretory IgA in the saliva is essential for protection from mucosally transmitted pathogens and maintaining homeostasis at mucosal surfaces of the oral cavity. Expression of submandibular gland polymeric Ig receptor (pIgR) is essential for IgA secretion. In the present study, we investigated the influence of indigestible carbohydrates on IgA production in the salivary gland and saliva. Five-week-old rats were fed a fibre-free diet (control), or a diet with $5 \%(\mathrm{w} / \mathrm{w})$ fructo-oligosaccharide (FOS) or a combination of $2.5 \%$ (w/w) polydextrose $(\mathrm{PDX})$ and $2.5 \%(\mathrm{w} / \mathrm{w})$ lactitol for 21-d. IgA concentrations in the caecal digesta, submandibular gland tissue, and saliva in the FOS and PDX + lactitol diet groups were significantly higher than those in the control group $(P<0 \cdot 05)$. The increase in IgA in the submandibular gland tissue was confirmed using immunohistochemical analysis. However, the IgA concentrations of serum did not differ between the FOS or PDX + lactitol groups and the control group $(P=0 \cdot 5)$. In the FOS and PDX + lactitol groups, the $p \operatorname{Ig} R$ mRNA $(p \operatorname{Ig} R / \beta$-actin) expression level in the submandibular gland tissue was significantly higher than that in the control group $(P<0 \cdot 05)$. The present study suggests that indigestible carbohydrates play an important role in the increase in IgA concentrations in the submandibular gland tissue, saliva, and caecal digesta.

Key words: IgA: Polymeric Ig receptor: Salivary gland: Saliva

The intestinal epithelium is the largest mucosal surface in the human body, and is constantly exposed to potentially toxic environmental antigens, pathogenic food and water-borne micro-organisms and commensal microflora ${ }^{(1)}$. Dietary approaches to influence human gut have long been used as an approach to improve host health ${ }^{(2)}$. Indigestible carbohydrates have beneficial effects in human health and nutrition ${ }^{(3)}$. Human digestive enzymes have little or no effect on carbohydrates such as fructo-oligosaccharides (FOS) and polydextrose (PDX). These substances are digested by colonic bacteria with the production of organic acids, mainly SCFA and gas ( $\mathrm{CO}_{2}$ and hydrogen). Small amounts of lactic, formic and succinic acids are also produced ${ }^{(4)}$. These health effects include immune regulation, normalization of blood glucose and insulin levels, and prevention of cancer recurrence ${ }^{(5)}$. Many indigestible carbohydrates influence aspects of intestinal function through fermentation ${ }^{(6)}$.
The use of indigestible carbohydrates results in increased IgA levels in the caecal digesta and faeces of animals ${ }^{(7-11)}$. In human subjects, IgA is the most abundant Ig isotype in the body in the absence of infection. It is synthesized mainly in the secretory form in gut-associated lymphoid tissues. Secretory IgA (sIgA) prevents pathogens and commensal bacteria from binding to the epithelial cells of the mucosa and neutralizes their toxins to maintain homeostasis at mucosal surfaces ${ }^{(12)}$. The polymeric Ig receptor (pIgR), also known as membrane secretory component, is an integral membrane protein expressed by intestinal epithelial cells. The physiological role of $\mathrm{pIgR}$ is to bind and transport J-chain-containing dimeric/polymeric IgA or polymeric IgM antibodies across the intestinal epithelial cell layer, and to protect them from proteolytic degradation in the secretions ${ }^{(13)}$. Furthermore, $\operatorname{PIg} R$ expression is increased by FOS in the intestines of infant mice ${ }^{(6,14)}$. Thus, IgA and pIgR play a very important

Abbreviations: FOS, fructo-oligosaccharides; FOS diet, 5\% fructo-oligosaccharide; pIgR, polymeric Ig receptor; PDX, polydextrose; PDX + lactitol diet, combination of $2.5 \%$ polydextrose and $2.5 \%$ lactitol; sIgA, secretory IgA.

*Corresponding author: Dr K. Tsukinoki, fax +81 46822 8866, email tsukinoki@kdu.ac.jp 
role in intestinal immunity influenced by indigestible carbohydrates.

The oral cavity is protected by the mucosal immune system ${ }^{(8)}$. The most important antibody class present in saliva is SIgA, which is produced as dimeric IgA by local plasma cells in the stroma of salivary glands, and is transported through the secretory epithelia by the $\operatorname{pIgR}^{(15)}$. Salivary sIgA plays a crucial role in mucosal immune function, and is the first line of defence in the gastrointestinal tract ${ }^{(16)}$. Rounds of heavy exercise depress salivary sIgA secretion, which results in an increased risk of infection, such as upper respiratory tract infection ${ }^{(17,18)}$. Children with no detectable IgA in their serum or whole saliva have been found to develop frequent bronchopulmonary infections, bronchial asthma, gastrointestinal allergy, and other conditions ${ }^{(19)}$. IgA plays important roles in the health of the oral cavity and upper respiratory tract. However, there are no reports regarding the relationship between IgA and pIgR and indigestible carbohydrates in the salivary gland to our knowledge.

In the present study, we focused on indigestible carbohydrates, which can increase IgA concentrations in the intestinal $\operatorname{tract}^{(2,3,6,20-23)}$. We hypothesized that indigestible carbohydrates may increase $\operatorname{Ig} A$ expression not only in the intestinal tract but also in the salivary gland. Therefore, we examined alteration of IgA content in the salivary gland tissue and saliva. In addition, the $\operatorname{pIg} R$ expression level was investigated in the salivary gland.

\section{Materials and methods}

\section{Animals}

Male Wistar rats ( 4 weeks old) were purchased from Japan CLEA and housed in wire mesh cages without bedding material, under controlled temperature $\left(22 \pm 3^{\circ} \mathrm{C}\right)$ and a $12 \mathrm{~h}$ light $-12 \mathrm{~h}$ dark cycle. The rats had free access to a commercial diet (CE-2; Japan CLEA) and water for $7 \mathrm{~d}$ before starting the experiment. The experimental protocol used in this study was reviewed and approved by the Ethics Committee for Animal Experiments of Kanagawa Dental University (approval number 20130613) and was performed in accordance with the Guidelines for Animal Experimentation of Kanagawa Dental University and the ARRIVE guidelines for reporting animal research. In total, fifteen rats were used in Expt 1 and were randomly divided into three groups (see next paragraph on 'Diets'). One cage held two or three rats. In Expt 2, eighteen rats were used and divided into three groups at random. For this experiment, three rats were kept in each cage. We maintained the rats in each group under the same conditions other than diet during the experiment period, and no rats died during the experiment. The rats had free access to diet and water. All rats were killed between 07.00 and 11.00 hours; they were first anaesthetized by pentobarbital anaesthesia, then blood was drawn by cardiac puncture, and they were then decapitated.

\section{Diets}

The composition of the fibre-free control diet and experimental diets are shown in Table 1 . The control diet was
Table 1. The composition (\%) of control and experimental diets

\begin{tabular}{lccc}
\hline & \multicolumn{3}{c}{ Diet groups } \\
\cline { 2 - 4 } Ingredient & Control & FOS & PDX + lactitol \\
\hline Casein & 20.0 & 20.0 & 20.0 \\
Sucrose & 70.0 & 65.0 & 65.0 \\
Maize oil & 5.00 & 5.00 & 5.00 \\
AIN76 M-Mix & 3.50 & 3.50 & 3.50 \\
AIN76 V-Mix & 1.00 & 1.00 & 1.00 \\
DL-Met & 0.300 & 0.300 & 0.300 \\
Choline bitartrate & 0.200 & 0.200 & 0.200 \\
Fructo-oligosaccharide & - & 5.00 & - \\
Polydextrose & - & - & 2.50 \\
Lactitol & - & - & 2.50 \\
Total (\%) & 100 & 100 & 100 \\
\hline
\end{tabular}

FOS, fructo-oligosaccharides; PDX, polydextrose.

based on the solidified AIN76 formulation, with all maize starch and cellulose were replaced by sucrose. FOS (Meioligo $\mathrm{P}^{\circledR}$; Meiji Food Material Company Limited) was added at $5 \%(\mathrm{w} / \mathrm{w})$ for the FOS diet. PDX (Litesse Ultra; Danisco Japan Limited) and lactitol (lactitol MC; Danisco Japan Limited) were each added at $2.5 \%(\mathrm{w} / \mathrm{w})$ for the PDX + lactitol diet. These special diets were made by Japan CLEA (Tokyo Japan). We employed AIN76 to eliminate the flux of carbohydrates such as maize starch and cellulose, which also induce production of SCFA ${ }^{(24,25)}$ in the large intestine. Sucrose is absorbed in the small intestine and does not reach the large intestine; thus, sucrose does not influence fermentation. Consumption of AIN76 results in lower levels of SCFA in the faeces of rats, compared with consumption of AIN93, which is based on maize starch ${ }^{(26)}$. Thus, the diets used in our study can reduce the influence of SCFA generated from maize starch, thereby allowing the influence of the fermentation of FOS, PDX and lactitol to be determined. On average, approximately $25 \mathrm{~g}$ of the diet was fed to each rat per $\mathrm{d}$. We eliminated any effect of the amount of the diet throughout the experiment.

\section{Sampling}

After $21 \mathrm{~d}$ of feeding, all rats were anaesthetised with sodium pentobarbital $(65 \mathrm{mg} / \mathrm{kg}$ body weight; Kyoritsu Seiyaku Corporation) for sampling. After deep anaesthesia of all rats, caecal digesta, submandibular glands, and blood samples were obtained in Expt 1. Saliva samples and submandibular glands were obtained in Expt 2. Blood samples were collected by cardiac puncture into Venoject II tubes containing Serum Separating Medium (Terumo). Tubes were immediately inverted five to six times and placed at room temperature for $3 \mathrm{~h}$. The samples were then centrifuged $(1200 \mathrm{~g}, 20 \mathrm{~min}$, $20^{\circ} \mathrm{C}$ ). All samples were stored at $-20^{\circ} \mathrm{C}$ until analysis. All samples were collected between 07.00 and 11.00 hours.

\section{Saliva collection}

Five minutes after anesthetisation, salivary secretion was elicited by intraperitoneal injection of pilocarpine $(8 \mathrm{mg} / \mathrm{kg}$ body weight; Nacalai Tesque, Inc.). After salivation began 
Table 2. Weight gain of rats during 21-d on experimental diets* (Mean values and standard deviations)

\begin{tabular}{|c|c|c|c|c|c|c|}
\hline \multirow[t]{2}{*}{ Group... } & \multicolumn{2}{|c|}{ Control } & \multicolumn{2}{|c|}{ FOS } & \multicolumn{2}{|c|}{ PDX + lactitol } \\
\hline & Mean & SD & Mean & SD & Mean & SD \\
\hline Expt $1(n 5)$ & $139 \cdot 6$ & $1 \cdot 7$ & 138.0 & $2 \cdot 8$ & $136 \cdot 4$ & $2 \cdot 2$ \\
\hline Expt $2(n 6)$ & 137.0 & $5 \cdot 0$ & $135 \cdot 7$ & 4.4 & $134 \cdot 2$ & 1.6 \\
\hline
\end{tabular}

FOS, fructo-oligosaccharides; PDX, polydextrose.

${ }^{*}$ No significant differences in the mean body weight gain between the control and FOS or PDX + lactitol groups (Expt 1, $P=0.5$; Expt 2, $P=0.9$; one-way ANOVA).

(approximately $5 \mathrm{~min}$ after injection), whole saliva was collected by pipette for $10 \mathrm{~min}$. All saliva samples were stored at $-20^{\circ} \mathrm{C}$ until analysis.

\section{Measurement of IgA concentration}

The concentration of IgA in the caecal digesta, right submandibular glands, serum, and saliva of rats was quantified by an ELISA using a Rat IgA ELISA Quantitation Set (Bethyl Laboratories).

Caecal digesta samples were treated with twenty volumes of distilled water with $1 \mathrm{~mm}$-phenylmethylsulphonyl fluoride from dimethyl sulphoxide-dissolved stock for $60 \mathrm{~min}$ at room temperature. The samples were then centrifuged $(10000 \mathrm{~g}$, $15 \mathrm{~min}, 4^{\circ} \mathrm{C}$ ) and the supernatant fractions were used for IgA measurement.

Submandibular gland tissue samples were crushed using a Cryo-press (Microtec Company Limited) and mixed in PBS (0.01 M, pH 7.2-7.4; Wako Pure Chemical Industries) containing $1 \%$ Triton $^{\circledR}$ X-100 (MP Biomedicals, LLC) and 1 mm-phenylmethylsulphonyl fluoride (from dimethyl sulphoxide-dissolved stock). These solutions were centrifuged $(10000 \mathbf{g}, 15 \mathrm{~min}$, $4^{\circ} \mathrm{C}$ ). The supernatant fractions were collected.

Ninety-six-well microtitre plates were coated for $1 \mathrm{~h}$ at room temperature $\left(20-25^{\circ} \mathrm{C}\right)$ with goat anti-rat $\operatorname{IgA}(1: 100$ dilution $)$ diluted with the coating buffer, $0.05 \mathrm{M}$-carbonate-bicarbonate, $\mathrm{pH}$ 9.6. The residual fluid was removed and washed five times with wash solution $(50 \mathrm{~mm}$-Tris, $0 \cdot 14 \mathrm{M}-\mathrm{NaCl}$, $0.05 \%$ Tween 20, pH 8.0). Blocking solution (50 mm-Tris, $0 \cdot 14 \mathrm{M}-\mathrm{NaCl}, 1 \%$ bovine serum albumin $\mathrm{pH} 8.0$ ) was added to the wells as a blocking agent. The plate was then incubated for $30 \mathrm{~min}$ at room temperature and washed with wash solution as before. Samples and rat IgA standards (Bethyl Laboratories) were added to each well. The plate was incubated for $1 \mathrm{~h}$ at room temperature and washed five times with wash solution. Horseradish peroxidase-conjugated goat anti-rat IgA detection antibody (1:15000 dilution) was added to each well. The plate was incubated again for $1 \mathrm{~h}$ at room temperature. The enzyme substrate $3,3^{\prime}, 5,5^{\prime}$-tetramethylbenzidine was added to each well after washing. The plate was developed in the dark at room temperature for $15 \mathrm{~min}$. The reaction was stopped with $0 \cdot 18 \mathrm{M}-\mathrm{H}_{2} \mathrm{SO}_{4}$ stop solution. Absorbance was measured at a wavelength of $450 \mathrm{~nm}$ in an automated microplate reader (BioRad). Absolute concentrations $(\mathrm{ng} / \mathrm{ml})$ were calculated using a standard curve. The IgA flow rate $(\mathrm{ng} / \mathrm{min})$ was calculated by multiplying the absolute concentration of IgA by saliva flow rate $(\mathrm{ml} / \mathrm{min})$.
The IgA flow rate was employed as an indicator of the amount of IgA stored in the salivary glands. The saliva flow rate was determined by dividing the weight of saliva with the sampling time assuming that the specific gravity of saliva was 1.00 .

\section{RNA extraction and complementary DNA synthesis}

Total RNA isolation from the submandibular glands was performed using ISOGEN reagent (Nippon Gene) in accordance with the manufacturer's instructions. RNA quality was determined by the ribosomal RNA banding pattern after electrophoresis on a $1.5 \%$ agarose gel containing ethidium bromide and visualization using UV illumination. RNA concentrations were determined using a Bio Spec-nano spectrophotometer (Shimadzu Access Corporation). Complementary DNA was synthesized from total RNA using a first-strand cDNA synthesis kit (Roche Diagnostics Limited).

\section{Quantitative real-time $P C R$ measurement of polymeric Ig receptor (plgR) mRNA in submandibular glands}

Real-time PCR was performed using a LightCycler 480 system (Roche Diagnostics Limited) according to the manufacturer's instructions. Reactions were performed in a $20-\mu l$ volume ( $0.5 \mu \mathrm{mol}$ each primer, $0.1 \mu \mathrm{mol}$ TaqMan Probe). The primer sequences used to amplify the $p \operatorname{Ig} R$ gene sequence were 5'-TGG GAG CTA CAA GTG TGG TC-3' (forward primer) and $5^{\prime}$-GGG TGT CAT TTG GGA ATC CAG-3' (reverse primer). The probe sequences were FAM- $5^{\prime}$-TTC GAT GTC AGC CTG GAG GTC AGC-3'-TAMRA TaqMan Probe designed and synthesized by Nippon Gene Research Laboratory. The PCR product was $98 \mathrm{bp}$. PCR amplification of pIgR was performed as follows: $95^{\circ} \mathrm{C}$ for $10 \mathrm{~min}$, followed by forty-five cycles of $95^{\circ} \mathrm{C}$ for $10 \mathrm{~s}$ and $62^{\circ} \mathrm{C}$ for $30 \mathrm{~s}$. Real-time PCR for amplification of the rat $\beta$-actin housekeeping gene was performed using a LightCycler, FastStart DNA Master SYBR Green I (Roche Diagnostics Limited), forward primer (5'-CTT GTA TGC CTC TGG TCG TA- $3^{\prime}$ ), and reverse primer (5'-CCA TCT CTT GCT CGA AGT CT- $3^{\prime}$ ) in accordance with the manufacturer's instructions (Nihon Gene Research Labs, Inc.).

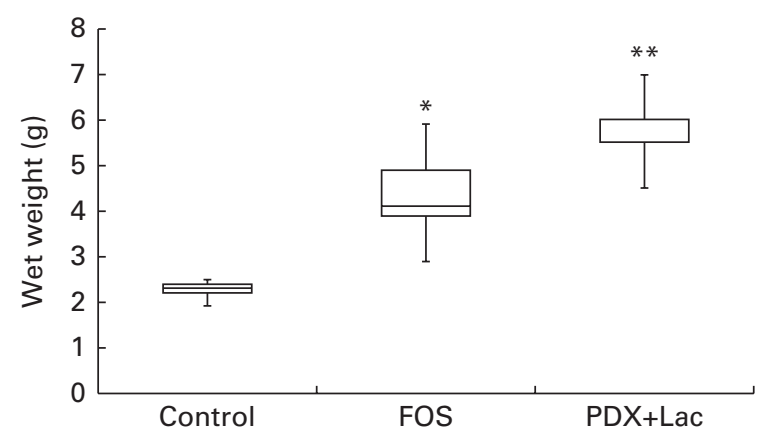

Fig. 1. Wet weight of caecal digesta $(n 5)$. Box plots show median, first quartile, third quartile, minimum and maximum values. Significant differences were determined using the Kruskal-Wallis test and multiple comparisons using the Steel test. Median value was significantly different from that of the control group: ${ }^{*} P<0.05$, ${ }^{\star *} P<0.01$. FOS, $5 \%$ fructo-oligosaccharide; PDX + Lac, combination of $2.5 \%$ polydextrose and $2.5 \%$ lactitol. 


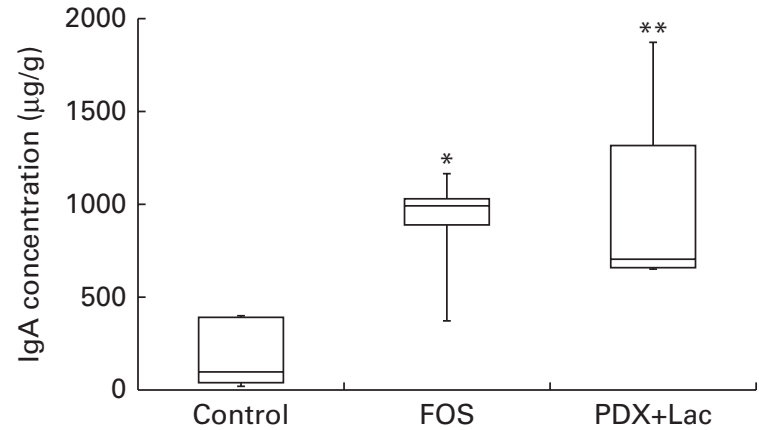

Fig. 2. IgA concentrations in caecal digesta ( $n$ 5). Box plots show median, first quartile, third quartile, minimum and maximum values. Significant differences were determined using the Kruskal-Wallis test and multiple comparisons using the Steel test. Median value was significantly different from that of the control group: ${ }^{\star} P<0.05$, ${ }^{\star \star} P<0.01$. FOS, $5 \%$ fructo-oligosaccharide; PDX + Lac, combination of $2.5 \%$ polydextrose and $2.5 \%$ lactitol.

The PCR product was $260 \mathrm{bp}$. Denaturation was performed at $95^{\circ} \mathrm{C}$ for $10 \mathrm{~min}$, followed by forty cycles of $95^{\circ} \mathrm{C}$ for $10 \mathrm{~s}, 60^{\circ} \mathrm{C}$ for $10 \mathrm{~s}$, and $72^{\circ} \mathrm{C}$ for $10 \mathrm{~s}$. Gene expression is stated in terms of the copy number ratio of $\operatorname{pIg} R$ mRNA: $\beta$-actin mRNA for each sample.

\section{Tissue preparation for immunohistochemistry}

Submandibular gland tissue samples were fixed in $4 \%$ buffered paraformaldehyde ( $\mathrm{pH} 7 \cdot 4$; Wako Pure Chemical Industries) for $24 \mathrm{~h}$ and embedded in paraffin. Serial $4-\mu \mathrm{m}$ sections were cut and stained with haematoxylin and eosin for immunohistochemistry. Immunohistochemical analysis was performed using simple stain rat MAX-PO (Nichirei Biosciences, Inc.). Slides were placed in $10 \mathrm{~mm}$-sodium citrate, $\mathrm{pH} 6 \cdot 0$, and microwaved for $20 \mathrm{~min}$ for antigen retrieval. Then $0 \cdot 2 \%$ Triton $^{\circledR} \mathrm{X}-100$ (MP Biomedicals, LLC) treatment was performed for $10 \mathrm{~min}$. Slides were pre-incubated in $3 \%$ $\mathrm{H}_{2} \mathrm{O}_{2}$ in methanol for $15 \mathrm{~min}$. Sections were incubated with anti-rat IgA rabbit polyclonal antibody (1:300; Abbiotec, LLC) for $2 \mathrm{~h}$ at room temperature. After washing with PBS, sections were incubated with the secondary antibody, horseradish peroxidase-labelled anti-rabbit IgG with amino acid polymer (Nichirei Biosciences, Inc.), for $30 \mathrm{~min}$ at room temperature. Colour was developed using 0.02\% 3,3'-diaminobenzidinetetrahydrochloride (Wako Pure Chemical Industries Limited) and $0.0003 \% \mathrm{H}_{2} \mathrm{O}_{2}$ in Tris-buffered saline for $6 \mathrm{~min}$. Sections were then counter-stained with hematoxylin. Non-immunised rabbit IgG was used instead of primary antibody for negative controls.

\section{Statistical analysis}

Statistical analyses were performed using SPSS software, version 17.0 (SPSS, Inc.). The statistical analyses for weight gain were performed using the one-way ANOVA. Other statistical analyses were performed using the Kruskal-Wallis test. If the results of the statistical analyses were significant, multiple comparisons were conducted using the Steel test. $P<0.05$ was considered statistically significant.

\section{Results}

Weight gain and caecal digesta weight of rats on fructo-oligosaccharide or polydextrose + lactitol diets

There were no significant differences in the intake of experimental diets between the control and FOS or PDX + lactitol groups. Prior to the experimental period, all rats had similar body weights. No significant differences were observed throughout the duration of the experimental period in mean body weight gain between the control and FOS or PDX + lactitol groups (Expt 1, $P=0.5$; Expt 2, $P=0 \cdot 9$ ) (Table 2). FOS and PDX + lactitol groups exhibited significantly increased caecal digesta wet weight compared to that of the control group (Expt 1, $P<0 \cdot 05$, Fig. 1).

\section{IgA concentrations of rat caecal digesta, submandibular gland tissue, and serum}

There were significant differences between the control group and both the FOS and the PDX + lactitol groups (Expt 1, $P<0.05)$ with respect to the IgA concentration in the caecal digesta after the 21-d feeding period (Fig. 2). Similarly, the IgA concentrations of the submandibular gland tissue were significantly increased in the FOS and PDX + lactitol groups compared to those of the control group (Expt 1, $P<0.05$, Fig. 3). However, the IgA concentrations of serum did not differ between groups (Expt 1, $P=0 \cdot 5$, Fig. 4).

\section{Immunohistochemical analysis of rat submandibular gland tissue}

After the 21-d feeding period in Expt 1, the submandibular glands of the rats were removed and processed for immunohistochemistry to detect IgA. No apparent signal for IgA was identified in the control group (Fig. 5(a)). IgA staining was observed in the acinar cells but not in ductal cells in the FOS (Fig. 5(b)) and PDX + lactitol (Fig. 5(c)) groups. Acinar cells exhibited weak cytoplasmic staining for IgA. In the stroma around the acinar cells, plasma cells were not recognized on light microscopy of haematoxylin and eosin staining

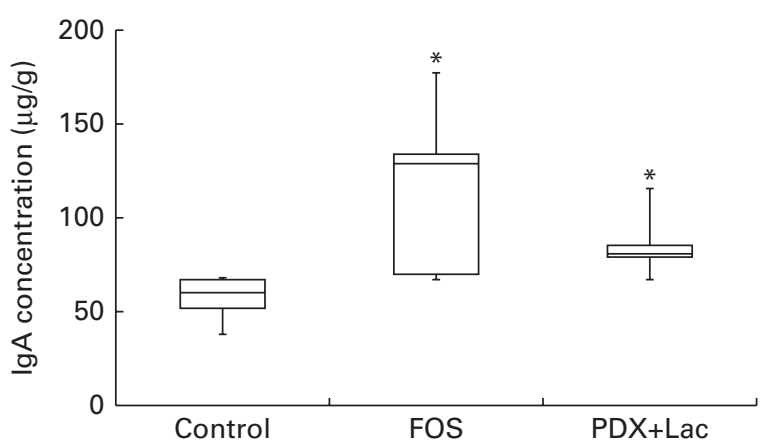

Fig. 3. IgA concentrations in submandibular gland tissue $(n 5)$. Box plots show median, first quartile, third quartile, minimum and maximum values. Significant differences were determined using the Kruskal-Wallis test and multiple comparisons using the Steel test. * Median value was significantly different from that of the control group $(P<0.05)$. FOS, $5 \%$ fructo-oligosaccharide; PDX + Lac, combination of $2.5 \%$ polydextrose and $2.5 \%$ lactitol. 


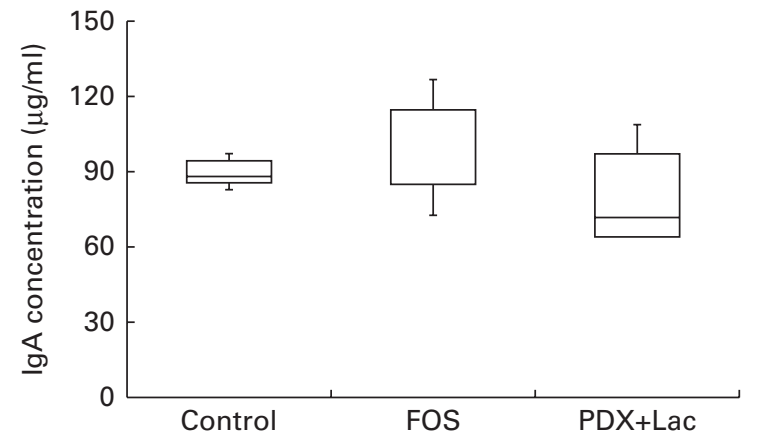

Fig. 4. Serum IgA concentrations ( $n$ 5). Box plots show median, first quartile, third quartile, minimum and maximum values. No significant differences in the median IgA concentration between the control and fructo-oligosaccharide (FOS) or combination of $2.5 \%$ polydextrose and $2.5 \%$ lactitol (PDX + Lac) groups ( $P=0.5$; Kruskal-Wallis test).

(data not shown) and immunohistochemical staining (see figures), although plasma cells may still have been present. Therefore, IgA-positive plasma cells were not observed in the submandibular glands of any group. Negative control slides were negative for IgA staining (data not shown).

\section{Saliva flow rate, IgA concentration, and IgA flow rate} of rat saliva

The saliva flow rate of rats, as measured for $10 \mathrm{~min}$, did not show significant differences between the control and FOS or PDX + lactitol groups with the Kruskal-Wallis test (Expt 2, $P=0 \cdot 4)$. The IgA concentrations in saliva from the FOS and PDX + lactitol groups were significantly increased compared to those from the control group (Expt 2, $P<0.05$, Fig. 6)
The IgA flow rate was significantly increased in the FOS and PDX + lactitol groups compared to that in the control group (Expt 2, $P<0 \cdot 01$, Fig. 7).

\section{Expression level of polymeric Ig receptor (plgR) mRNA in submandibular gland tissue}

Quantitative PCR was conducted on complementary DNA samples as described in the methods to determine the level of pIgR transcript accumulation in the submandibular glands of the rats after the feeding period. Melting curve analysis revealed a single fluorescence peak representing the $T_{\mathrm{m}}$ of the $\mathrm{pIgR}$ PCR product in all samples. The negative sample did not yield a product (data not shown). A single band was observed following agarose gel electrophoresis of the PCR product from all samples (data not shown). In FOS and PDX + lactitol groups, the $p I g R$ mRNA ( $p I g R / \beta$-actin) expression level in the submandibular gland tissue was significantly increased compared to that of control group (Expt 2, $P<0 \cdot 05$, Fig. 8).

\section{Discussion}

It has been reported that intake of indigestible carbohydrates such as FOS, PDX, and lactitol increases the IgA concentration in the rat caecal digesta ${ }^{(7,27-30)}$. Accordingly, in the present study, addition of FOS or a combination of lactitol and PDX caused significant elevation of IgA concentration in the caecal digesta of rats (Fig. 2). We used an AIN76-modified diet with all fibre components eliminated as a control group, and only FOS or a combination of PDX and lactitol was added to the experimental groups. This model minimizes the influence of starch and enables isolation of the effect of
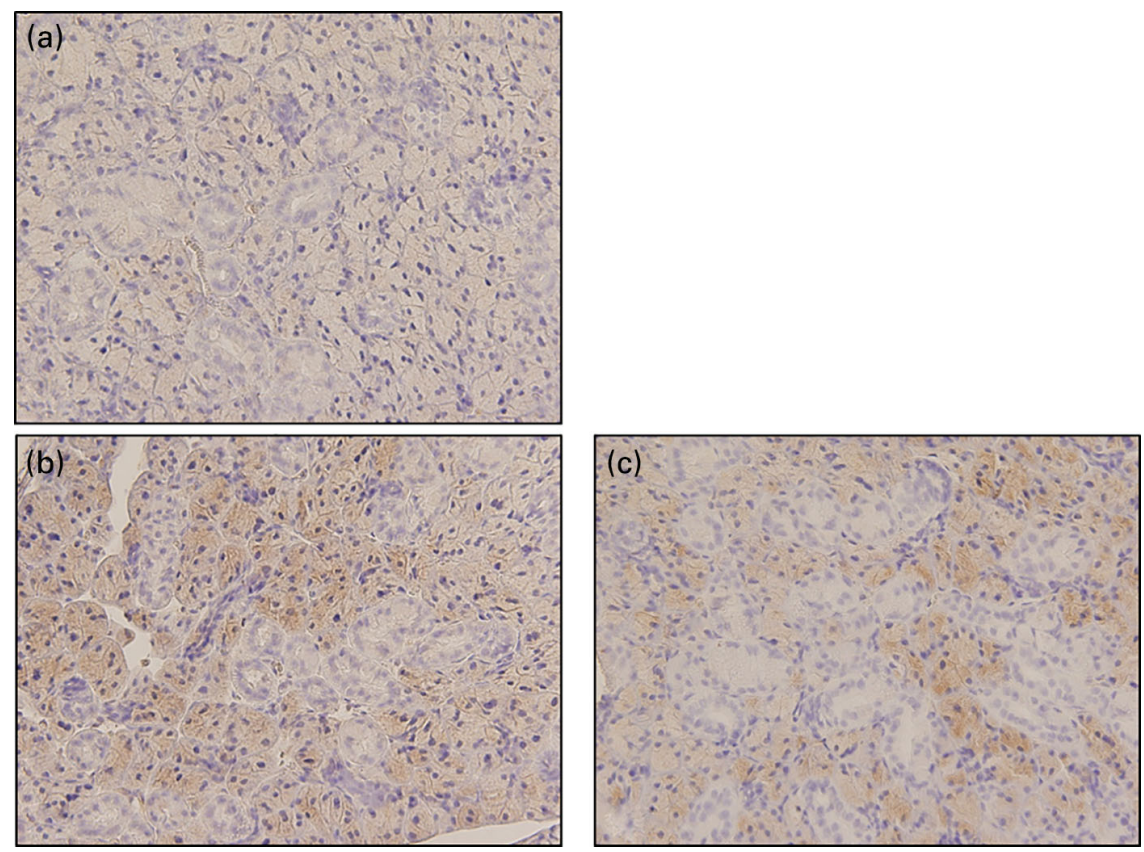

Fig. 5. Immunohistochemical analysis of IgA protein in submandibular gland tissue. Control (a), fructo-oligosaccharide (FOS) (b) or combination of $2.5 \%$ polydextrose and $2.5 \%$ lactitol (PDX + Lac) (c). IgA immunohistochemical signals showed positive reaction for FOS or PDX + Lac group, compared with the control group. Magnification (400x). 


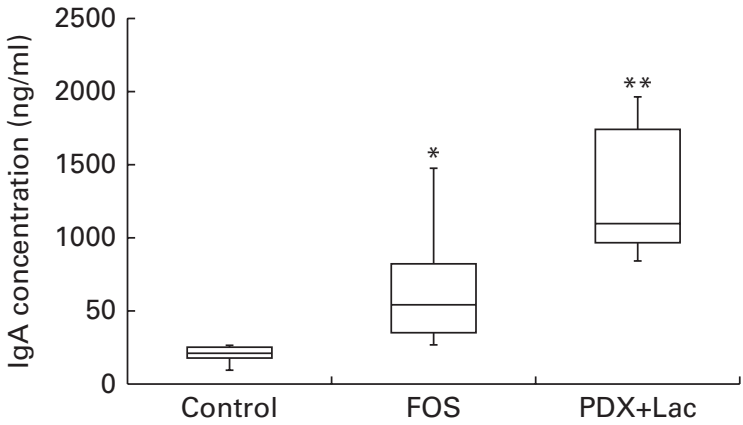

Fig. 6. Saliva IgA concentrations ( $n 6$ ). Box plots show median, first quartile, third quartile, minimum and maximum values. Significant differences were determined using the Kruskal-Wallis test and multiple comparisons using the Steel test. Median value was significantly different from that of the control group: ${ }^{*} P<0.05,{ }^{* \star} P<0.01$. FOS, $5 \%$ fructo-oligosaccharide; PDX + Lac, combination of $2.5 \%$ polydextrose and $2.5 \%$ lactitol.

PDX, lactitol, and FOS. We also evaluated the IgA concentration in the salivary glands and saliva. Consequently, the present study demonstrates the influence of these carbohydrates on salivary IgA production.

In our model, IgA concentration in the submandibular gland was significantly increased by FOS or PDX + lactitol (Fig. 3). To our knowledge, this is the first report of submandibular gland IgA production induced by these carbohydrates. In addition, this model demonstrated that serum IgA concentrations did not increase upon intake of FOS or PDX + lactitol (Fig. 4). Delgado et al. ${ }^{(15)}$ reported that FOS derived from Yacón did not elevate the serum IgA level of mice. No changes in serum IgA were observed in healthy elderly people who were given indigestible carbohydrates ${ }^{(31)}$. Since IgA targets antigens at the mucosal surface, it is thought that an increase of IgA production in the submandibular gland and saliva does not affect serum concentrations of IgA. Interestingly, IgA staining was increased in the acinar cells of the submandibular gland upon FOS or PDX + lactitol intake, compared with that of the control diet. Because the IgA concentration in the submandibular gland was independent of serum IgA, the observed increases in IgA expression were apparently caused by increases in production in the local organ.

FOS and PDX + lactitol increased the IgA concentration in the saliva and submandibular gland and the IgA flow rate in the saliva compared with those of the control group (Figs. 3, 6 and 7). The IgA flow rate was employed as an indicator of the amount of IgA stored in the salivary glands. Ingestion of indigestible carbohydrates may increase the IgA concentration in the saliva and submandibular glands and the amount of IgA stored in the salivary glands, which may protect against infection by adventitious bacteria and viruses, e.g. upper respiratory tract infection ${ }^{(17,18)}$

Indigestible carbohydrates such as FOS, PDX and lactitol reach the large intestine, and then are degraded by the microflora. During such degradation SCFA and gases are formed ${ }^{(32)}$. In the present study, addition of FOS or PDX and lactitol increased SCFA production in the large intestine as in previous studies $^{(33-39)}$. FOS, PDX, and lactitol could theoretically also be fermented by bacteria in the oral cavity, which could be associated with dental caries. Generally, dental caries is associated with a loss of minerals such as $\mathrm{Na}$ and $\mathrm{Ca}$ under low $\mathrm{pH}$ in the dental plaque on tooth surfaces ${ }^{(40)}$. The escape of minerals from the tooth surface usually leads to the creation of carious cavities on the tooth surface. The low $\mathrm{pH}$ in dental plaque results from fermentation of monosaccharides and disaccharides by Streptococcus mutans. However, the retention period should probably be too short for $S$. mutans to ferment indigestible carbohydrates such as FOS, PDX and lactitol in the oral cavity. Indeed, it has been shown that $S$. mutans rarely degrades indigestible carbohydrates in the oral cavity ${ }^{(41)}$. Therefore, indigestible carbohydrates such as FOS, PDX and lactitol would probably not induce dental caries as in a previous study which did not show dental caries formation ${ }^{(42)}$. Adverse effects of indigestible carbohydrates such as FOS, PDX and lactitol include diarrhoea ${ }^{(43)}$; however, a smaller amount of indigestible carbohydrates was previously shown not to induce diarrhoea ${ }^{(43)}$. Addition of indigestible carbohydrates such as FOS, PDX and lactitol to the diet with careful consideration of the amounts added may thus reduce upper respiratory tract infection without diarrhoea.

The expression of $P I g R$ mRNA in the rat submandibular gland tissue and saliva IgA concentration were significantly increased upon intake of FOS or PDX + lactitol (Figs. 6 and 8). Nakamura et $a{ }^{(6,14)}$ reported that $p \operatorname{Ig} R$ expression increased in the intestinal tract upon indigestible carbohydrate intake. And pIgR plays an important role in facilitating the transport of IgA across the epithelial border and in the resistance to the IgA proteases present within mucosal secretions. Suppression of $\operatorname{PIg} R$ expression caused a decrease in the quantity of $\operatorname{sIgA}$ secreted within the saliva in a previous study ${ }^{(44)}$. Therefore, $\operatorname{pIg} R$ expression in the submandibular gland may cause an increase in IgA concentration and IgA flow rate in saliva.

Carpenter et al. ${ }^{(45)}$ reported that the rate of IgA secretion into saliva is regulated by the autonomic nerves supplying the glands in vivo. Following in vivo nerve stimulation, there was an increased amount of pIgR expressed on the membrane surface. This was also functionally demonstrated in vitro by increased uptake of human IgA by acutely prepared rat salivary cells following stimulation by adrenaline,

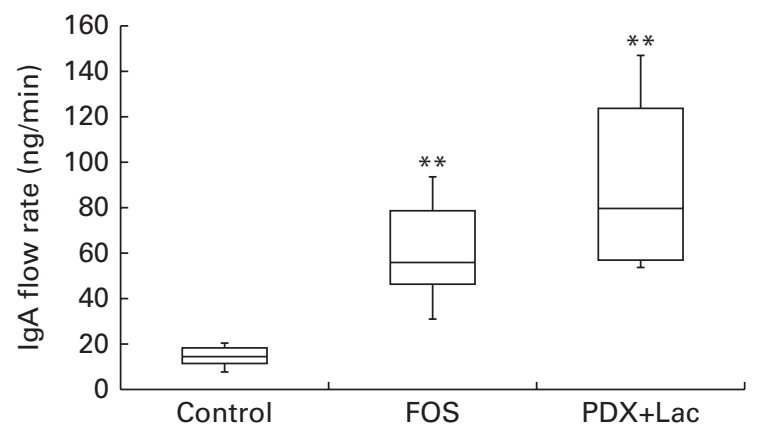

Fig. 7. IgA flow rate in saliva ( $n$ 6). Box plots show median, first quartile, third quartile, minimum and maximum values. Significant differences were determined using the Kruskal-Wallis test and multiple comparisons using the Steel test. ${ }^{* *}$ Median value was significantly different from that of the control group $(P<0.01)$. FOS, $5 \%$ fructo-oligosaccharide; PDX + Lac, combination of $2.5 \%$ polydextrose and $2.5 \%$ lactitol. 


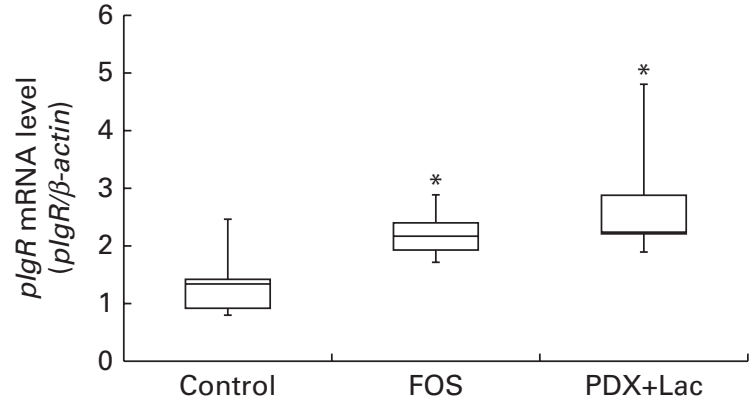

Fig. 8. Expression level of polymeric Ig receptor ( $p / g R$ ) mRNA in submandibular gland tissue $(n 6)$. Box plots show median, first quartile, third quartile, minimum and maximum values. Significant differences were determined using the Kruskal-Wallis test and multiple comparisons using the Steel test. ${ }^{*}$ Median value was significantly different from that of the control group $(P<0.05)$. FOS, $5 \%$ fructo-oligosaccharide; PDX + Lac, combination of $2.5 \%$ polydextrose and $2.5 \%$ lactitol.

indicating increased mobilisation of pIgR with stimulation ${ }^{(45)}$ Kimura et al. ${ }^{(46-48)}$ recently reported that two orphan G-protein coupled receptors (GPCR), GPR41 and GPR43, were most abundantly expressed in sympathetic ganglia in adipose tissue, the gut and the peripheral nervous system. These receptors have been reported to be activated by SCFA $^{(46-48)}$, which have been shown to be produced by intestinal bacterial fermentation of indigestible carbohydrates ${ }^{(4)}$. Among SCFA, butyrate was previously shown to induce the differentiation of $\mathrm{T}$ regulatory cells in vitro and in vivo, and to ameliorate the development of colitis induced by adoptive transfer of CD4(+) CD45RB(hi) T cells in Rag1(-/-) mice ${ }^{(49)}$. SCFA affect remote organs ${ }^{(50)}$, and the salivary glands are greatly influenced by autonomic nerve ${ }^{(51)}$. Therefore, further experiments are necessary to examine the influence of SCFA generated in the large intestine from indigestible carbohydrates, which may affect the submandibular gland and saliva IgA through the autonomic nervous system ${ }^{(45,52-54)}$.

In conclusion, we report the novel finding that indigestible carbohydrates in the diet increased submandibular gland IgA expression. Moreover, increases in local IgA production and pIgR expression may influence IgA concentration in the submandibular gland. These findings support the hypothesis that indigestible carbohydrates are important for the enhancement of oral mucosal immunity and of the caecal digesta.

\section{Acknowledgements}

The authors thank Professor Tatsuya Morita of Shizuoka University for technical advice on immunohistochemistry.

The present research was supported by the General Research Fund administered by Kanagawa Dental University under grant number 20130333. The funders had no role in the design, analysis or writing of this article.

The authors declare no conflicts of interest.

The author's contributions are as follows: Y. Y. collected all relevant literature, conducted all the experiments and statistical analyses, and wrote the first draft of the manuscript. M. T. performed all the experimental and statistical analyses. T. H. conducted ELISA analysis. T. S. attended to saliva collection. Y. K. performed the immunohistochemical analysis of rat submandibular gland tissue. J. S. conducted $p I g R$ mRNA analysis. T. T. designed the components of the fibre-free diet and participated in statistical analyses. K. T. designed the present study and wrote the first draft of the manuscript.

All authors contributed to, read and approved the final manuscript.

\section{References}

1. Baumann J, Park CG \& Mantisa NJ (2010) Recognition of secretory IgA by DC-SIGN: implications for immune surveillance in the intestine. Immunol Lett 131, 59-66.

2. Walton GE, Lu C, Trogh I, et al. (2012) A randomised, double-blind, placebo controlled cross-over study to determine the gastrointestinal effects of consumption of arabinoxylanoligosaccharides enriched bread in healthy volunteers. Nutr J 11, 36.

3. Velez E, Castillo N, Mesón O, et al. (2013) Study of the effect exerted by fructo-oligosaccharides from yacon (Smallanthus sonchifolius) root flour in an intestinal infection model with Salmonella Typhimurium. Br J Nutr 109, 1971-1979.

4. Mitsuoka T (2014) Development of functional foods. Biosci Microbiota Food Health 33, 117-128.

5. Kumar V, Sinha AK, Makkar HP, et al. (2012) Dietary roles of non-starch polysaccharides in human nutrition: a review. Crit Rev Food Sci Nutr 52, 899-935.

6. Nakamura Y, Nosaka S, Suzuki M, et al. (2004) Dietary fructooligosaccharides up-regulate immunoglobulin A response and polymeric immunoglobulin receptor expression in intestines of infant mice. Clin Exp Immunol 137, 52-58.

7. Peuranen S, Tiihonen K, Apajalahti J, et al. (2004) Combination of polydextrose and lactitol affects microbial ecosystem and immune responses in rat gastrointestinal tract. $\mathrm{Br} \mathrm{J}$ Nutr 91, 905-914.

8. Yasui H, Nagaoka N, Miyake A, et al. (1992) Detection of Bifidobacterium strains that induce large quantities of IgA. Microb Ecol Health Dis 5, 155-162.

9. Takahashi T, Nakagawa E, Nara T, et al. (1998) Effects of orally ingested Bifidobacterium longum on the mucosal IgA response of mice to dietary antigens. Biosci Biotechnol Biochem 62, 10-15.

10. Frece J, Kos B, Svetec IK, et al. (2009) Symbiotic effect of Lactobacillus belveticus M92 and prebiotics on the intestinal microflora and immune system of mice. J Dairy Res 76, 98-104.

11. Gourbeyre P, Desbuards N, Grémy G, et al. (2012) Exposure to a galactooligosaccharides/inulin prebiotic mix at different developmental time points differentially modulates immune responses in mice. J Agric Food Chem 60, 11942-11951.

12. Tezuka H, Abe Y, Asano J, et al. (2011) Prominent role for plasmacytoid dendritic cells in mucosal $\mathrm{T}$ cell-independent IgA induction. Immunity 34, 247-257.

13. Norderhaug IN, Johansen FE, Schjerven H, et al. (1999) Regulation of the formation and external transport of secretory immunoglobulins. Crit Rev Immunol 19, 481-508.

14. Nakamura Y, Terahara M, Iwamoto T, et al. (2011) Upregulation of polymeric immunoglobulin receptor expression by the heat-inactivated potential probiotic Bifidobacterium bifidum OLB6378 in a mouse intestinal explant model. Scand J Immunol 75, 176-183.

15. Delgado GT, Thomé R, Gabriel DL, et al. (2012) Yacon (Smallanthus sonchifolius)-derived fructooligosaccharides improves the immune parameters in the mouse. Nutr Res 32, 884-892.

16. Kotani1 Y, Shinkai S, Okamatsu H, et al. (2010) Oral intake of Lactobacillus pentosus strain b240 accelerates salivary 
immunoglobulin A secretion in the elderly: a randomized, placebo-controlled, double-blind trial. Immun Ageing 7, 11.

17. Otsuki T, Shimizu K, Iemitsu M, et al. (2012) Chlorella intake attenuates reduced salivary SIgA secretion in kendo training camp participants. Nutr J 11, 103.

18. Walsh NP, Gleeson M, Shephard RJ, et al. (2011) Position statement. Part one: immune function and exercise. Exerc Immunol Rev 17, 6-63.

19. Ostergaard PA (1980) Clinical and immunological features of transient IgA deficiency in children. Clin Exp Immunol 40, $561-565$

20. Scalabrin DM, Mitmesser SH, Welling GW, et al. (2012) New prebiotic blend of polydextrose and galacto-oligosaccharides has a bifidogenic effect in young infants. $J$ Pediatr Gastroenterol Nutr 54, 343-352.

21. Lecerf JM, Dépeint F, Clerc E, et al. (2012) Xylo-oligosaccharide (XOS) in combination with inulin modulates both the intestinal environment and immune status in healthy subjects, while XOS alone only shows prebiotic properties. Br J Nutr 28, 1847-1858.

22. Stuyven E, Van den Broeck W, Nauwynck H, et al. (2010) Oral administration of $\beta-1,3 / 1,6$-glucan Macrogard fails to enhance the mucosal immune response following oral $\mathrm{F} 4$ fimbrial immunisation in gnotobiotic pigs. Vet Immunol Immunopathol 137, 291-297.

23. Gouveia EM, Silva IS, Nakazato G, et al. (2013) Action of phosphorylated mannanoligosaccharides on immune and hematological responses and fecal consistency of dogs experimentally infected with enteropathogenic Escherichia coli strains. Braz J Microbiol 44, 499-504.

24. Frost GS, Walton GE, Swann JR, et al. (2014) Impacts of plantbased foods in ancestral hominin diets on the metabolism and function of gut microbiota in vitro. MBio 5, e00853-14.

25. Otles S \& Ozgoz S (2014) Health effects of dietary fiber. Acta Sci Pol Technol Aliment 13, 191-202.

26. Cresci A, Orpianesi C, Silvi S, et al. (1999) The effect of sucrose or starch-based diet on short-chain fatty acids and faecal microflora in rats. J Appl Microbiol 86, 245-250.

27. Yasuda A, Inoue K, Sanbongi C, et al. (2012) Dietary supplementation with fructooligosaccharides attenuates allergic peritonitis in mice. Biochem Biophys Res Commun 422, 546-550.

28. Fava1 F, Mäkivuokko H, Siljander-Rasi H, et al. (2007) Effect of polydextrose on intestinal microbes and immune functions in pigs. Br J Nutr 98, 123-133.

29. Watzl B, Girrbach S \& Roller M (2005) Oligofructose and immunomodulation. Br J Nutr 93, Suppl. 1, S49-S55.

30. Swanson KS, Grieshop CM, Flickinger EA, et al. (2002) Supplemental fructooligosaccharides and mannanoligosaccharides influence immune function, ileal and total tract nutrient digestibilities, microbial populations and concentrations of protein catabolites in the large bowel of dogs. J Nutr 132, 980-989.

31. Bunout D, Hirsch S, Pía de la Maza M, et al. (2002) Effects of prebiotics on the immune response to vaccination in the elderly. JPEN J Parenter Enteral Nutr 26, 372-376.

32. Nyman M (2002) Fermentation and bulking capacity of indigestible carbohydrates: the case of inulin and oligofructose. Br J Nutr 87, S163-S168.

33. Hiroki T, Hiroyuki I, Kimio S, et al. (2006) Dietary indigestible components exert different regional effects on luminal mucin secretion their bulk-forming property and fermentability. Biosci Biotechnol Biochem 70, 1188-1194.

34. Ito H, Wada T, Ohguchi M, et al. (2008) The degree of polymerization of inulin-like fructans affects cecal mucin and immunoglobulin A in rats. J Food Sci 73, H36-H41.
35. Moreau NM, Martin LJ, Toquet CS, et al. (2003) Restoration of the integrity of rat caeco-colonic mucosa by resistant starch, but not by fructo-oligosaccharides, in dextran sulfate sodium-induced experimental colitis. Br J Nutr 90, 75-85.

36. Beards E, Tuohy K \& Gibson G (2010) Bacterial, SCFA and gas profiles of a range of food ingredients following in vitro fermentation by human colonic microbiota. Anaerobe 16, 420-425.

37. Mäkeläinen HS, Mäkivuokko HA, Salminen SJ, et al. (2007) The effects of polydextrose and xylitol on microbial community and activity in a 4-stage colon simulator. J Food Sci $\mathbf{7 2}$, M153-M159.

38. Probert HM, Apajalahti JH, Rautonen N, et al. (2004) Polydextrose, lactitol, and fructo-oligosaccharide fermentation by colonic bacteria in a three-stage continuous culture system. Appl Environ Microbiol 70, 4505-4511.

39. Finney M, Smullen J, Foster HA, et al. (2007) Effects of low doses of lactitol on faecal microflora, $\mathrm{pH}$, short chain fatty acids and gastrointestinal symptomology. Eur J Nutr 46, 307-314.

40. Struzycka I (2014) The oral microbiome in dental caries. Pol J Microbiol 63, 127-135.

41. Sakallioğlu Ö, Güvenç IA \& Cingi C (2014) Xylitol and its usage in ENT practice. J Laryngol Otol 128, 580-585.

42. Zero DT (2008) Are sugar substitutes also anticariogenic? J Am Dent Assoc 139, Suppl., 9S-10S.

43. Oku T, Nakamura S \& Ichinose M (2005) Maximum permissive dosage of lactose and lactitol for transitory diarrhoea, and utilizable capacity for lactose in Japanese female adults. J Nutr Sci Vitaminol 51, 51-57.

44. Kimura F, Aizawa K, Tanabe K, et al. (2008) A rat model of saliva secretory immunoglobulin: a suppression caused by intense exercise. Scand J Med Sci Sports 18, 367-372.

45. Carpenter GH, Proctor GB \& Ebersole LE (2004) Secretion of IgA by rat parotid and submandibular cells in response to autonomimetic stimulation in vitro. Int Immunopharmacol 4, 1005-1014.

46. Kimura I, Inoue D, Maeda T, et al. (2011) Short-chain fatty acids and ketones directly regulate sympathetic nervous system via $G$ protein-coupled receptor 41 (GPR41). Proc Natl Acad Sci U S A 108, 8030-8035.

47. Inoue D, Tsujimoto G \& Kimura I (2014) Regulation of energy homeostasis by GPR41. Front Endocrinol (Lausanne) 5, 81.

48. Kimura I, Inoue D \& Hirano K (2014) The SCFA receptor GPR43 and energy metabolism. Front Endocrinol (Lausanne) 5, 85.

49. Furusawa Y, Obata Y, Fukuda S, et al. (2013) Commensal microbe-derived butyrate induces the differentiation of colonic regulatory T cells. Nature 19, 446-450.

50. Sakata T (1987) Stimulatory effect of short-chain fatty acids on epithelial cell proliferation in the rat intestine: a possible explanation for trophic effects of fermentable fibre, gut microbes and luminal trophic factors. Br J Nutr 58, 95-103.

51. Sabbadini E \& Berczi I (1995) The submandibular gland: a key organ in the neuro-immuno-regulatory network? Neuroimmunomodulation 2, 184-202.

52. Proctor GB, Carpenter GH \& Garrett JR (2000) Sympathetic decentralization abolishes increased secretion of immunoglobulin A evoked by parasympathetic stimulation of rat submandibular glands. J Neuroimmunol 109, 147-154.

53. Proctor GB, Garrett JR, Carpenter GH, et al. (2003) Salivary secretion of immunoglobulin A by submandibular glands in response to autonomimetic infusions in anaesthetised rats. J Neuroimmunol 136, 17-24.

54. Proctor GB \& Carpenter GH (2007) Regulation of salivary gland function by autonomic nerves. Auton Neurosci 133, $3-18$. 